\title{
Yield Response of Rice Genotypes for Gene Action under Coastal Saline Condition
}

\author{
R. Thirumalai*, K. Palaniraja and S. Vennila \\ Department of Genetics and Plant Breeding, Faculty of Agriculture, Annamalai University, \\ Annamalai Nagar, Tamil Nadu, India-608 002 \\ *Corresponding author
}

\begin{tabular}{|l|}
\hline Ke y w o r d s \\
Combining ability, \\
GCA, SCA, L $\times$ T, \\
Rice
\end{tabular}

\section{A B S T R A C T}

The present study, combining ability estimates were worked out through Line $\times$ Tester analysis of 32 hybrids developed by crossing eight lines with four testers to know the genetic architecture of 11 morphological traits under coastal saline condition. The present study was conducted at Plant Breeding Farm, Department of Genetic and Plant Breeding, Faculty of Agriculture, Annamalai University to estimate combining ability, gene action and proportional contribution of cross components in rice genotypes under coastal salinity. The analysis of variance of combining ability displayed variances of specific combining ability (SCA) were higher in magnitude than the corresponding general combining ability (GCA) variances for all the traits under study which indicated preponderance of nonadditive gene action governing these traits. Based on per se and gca effects the parents KULLAKAR, ASHWANI and ADT 39 among the lines and among the testers, IR 36, ADT 37 and ADT 4545 were found to be the best general combiner for grain yield and its component characters. The hybrids namely, KULLAKAR $\times$ IR 36, ASHWANI $\times$ ADT 37, ADT $39 \times$ ADT 45 and ASHWANI $\times$ ADT 45 were rated as superior crosses since they possessed high per se performance for most of the economic traits studied with earliness and short stature plant.

\section{Introduction}

Rice is one of the world most important staple food crops, feeding more than half of the world population. In rice crop salinity levels in soils are above thresholds affecting growth and yield. Recently, the salt affected area is increasing due to the irrigation with salt affected water, high intensity of cropping pattern and more application of chemical fertilizers. Unfortunately, rice is one of the most salt sensitive cereal crops. To meet the growing demand, more variability is required to develop new high yielding varieties with tolerance to salinity. Combining ability is a powerful tool in identifying the best combiners that may be used in crosses either to exploit heterosis or to accumulate fixable genes and obtain desirable segregates. It will help to understand the genetic architecture of various characters that enable the breeder to design effective breeding plan for future up gradation of the existing materials. This information may also be useful to breeders for 
genetic improvement of the existing genotypes on the basis of the performance in various hybrid combinations. The line $\mathrm{x}$ tester analysis in rice is used principally to determine the general and specific combining ability of the quantitative properties (Asadur Rahaman, 2016). Griffing (1956) stated that GCA includes the additive genetic portion, while SCA includes non-additive genetic portion of the total variation. The presence of non-additive genetic variance is the primary justification for initiating the hybrid programme (Cockerham, 1961; Pradhan and Singh et al, 2008). In this study, an attempt has been made to evaluate GCA and SCA in rice cultivars of different traits or identifying good combiners.

\section{Materials and Methods}

The experiment was conducted under coastal saline condition with EC of the soil ranged from 3.5 to $4.0 \mathrm{dSm}^{-1}$. Twelve selected genotypes of which eight genotypes (ADT 43, NLR 34449, AKSHYA, PONNI, ADT-39, KULLAKAR, ASHWANI and GIRI) were used as lines and three (ADT 37, ADT 45, IR 36 and IR 20) as testers. These parents were crossed to produce thirty two hybrids using line $\mathrm{x}$ tester mating design to the combining ability as well as gene action on yield and yield components. This study was conducted during July - October 2016 at plant breeding farm of Faculty of Agriculture, Annamalai University. One seedling per hill was transplanted at a spacing of $30 \mathrm{~cm}$ between rows and $20 \mathrm{~cm}$ within the rows in a randomized block design with three replications. Recommended cultural practices and need based plant protection measures were also adopted to raise the crop. Observations were recorded on ten competitive plants both in parents and hybrids in each replication for the following eleven traits viz., days to 50 per cent flowering, plant height at maturity, number of tillers per plant, number of productive tillers, panicle length, number of grains per panicle, kernel length, kernel breadth, kernel L/B ratio, hundred grain weight and grain yield per plant.

The statistical analysis was performed by using Line $\mathrm{x}$ Tester analysis by Kempthorne (1957) and genetic components of each parameter following Singh and Chaudhary (1985).

\section{Results and Discussion}

\section{Analysis of variance}

The analysis of variance showed significant variations for the yield and different yield contributing characters among the genotypes, crosses, lines, testers and line $\mathrm{x}$ tester interactions. The significant differences among the lines, testers and line $\mathrm{x}$ testers indicated that the genotype had wide genetic diversity among themselves for days to 50 per cent flowering, plant height at maturity, number of tillers per plant, number of productive tillers per plant, panicle length, number of grains per panicle, kernel length, kernel breadth, kernel L/B ratio, hundred grain weight and grain yield per plant. In combining ability, significant variances for lines and testers were observed for all the eleven characters. In case of lines $\times$ testers also significant positive variance was observed for all the characters.

The estimates of GCA and SCA variances revealed that the SCA variances had greater magnitude than GCA variance for all the characters studied, indicating the preponderance of non-additive gene action (Table 1). The significant differences between line $\times$ testers interaction indicates that SCA attributed heavily in the expression of these traits and demonstrates the importance of dominance or non additive variances for all the traits (Sanghera and Hussain, 2012). And 
also the variances of SCA were higher than the GCA variances for these traits which indicated preponderance of non additive gene action in the inheritance of traits. It suggested the greater importance of non-additive gene action in the expression and indicated very good prospect for exploitation of non additive genetic variation for yield and its component characters through hybrid breeding. The lines KULLAKAR, ASHWANI, and ADT 39 as well as in testers IR 36, ADT 37 and ADT 45 were observed to be good general combiners for most of the characters studied.

\section{General combining ability ( $g c a)$ effects}

Among the lines studied, KULLAKAR recorded the highest mean value for all the traits except kernel L/B ratio. Also, genotype KULLAKAR registered the earliness with minimum plant height. The line, ASHWANI also had significant high mean for all the traits except kernel L/B ratio. This genotype recorded significantly high mean value for hundred grain weight. Another line, ADT 39 showed significant high mean for all the characters except kernel length and kernel breadth the same genotype was founded to be earliest in duration. Among the testers, IR 36 recorded high per se performance for the traits viz., plant height, number of tillers per plant, number of productive tillers per plant, panicle length, grains per panicle, kernel length, hundred grain weight and grain yield per plant.

The tester, ADT 45 recorded significantly high mean performance for almost all the traits. Thus, based on the per se performance the lines, KULLAKAR, ASHWANI, and ADT 39 and the testers IR 36, ADT 45 and ADT 37 have been estimated as superior parents and they might be useful for the incorporation of the respective traits in hybridization programme (Table 2 ).

\section{Specific combining ability (sca) effects}

KULLAKAR $\times$ IR 36 and ASHWANI $\times$ ADT 37 had positive and highly significant sca effects for the traits viz., number of tillers per plant, number of productive tillers per plant, panicle length, number of grains per panicle, kernel length, kernel breadth, hundred grain weight and grain yield per plant along with negative significant sca effects for days to 50 $\%$ flowering and plant height. Similar results were obtained by Upadhyay and Jaiswal (2015) in their studies on rice.

The crosses, ADT $39 \times$ ADT 45 and ASHWANI $\times$ ADT 45 possessed high per se performance for most of the traits along with good performance for the plant height and days to $50 \%$ flowering. Also, these two hybrids had high $\mathrm{x}$ high combiners with non significant sca effects for grain yield and all its component traits with negative and non significant values for plant height and days to $50 \%$ flowering. This indicated that these crosses possessed favourable recombinants with short stature and earliness in later segregating generations. Similar results were found by Zeinab Montazer et al., (2014), Gayathri (2015) and Nainu et al., (2016) in their studies in rice (Table 3 ).

It is concluded, in the present study, KULLAKAR $\times$ IR 36, ASHWANI $\times$ ADT 37 , ADT $39 \times$ ADT 45 and ASHWANI $\times$ ADT 45 are identified as good general combiner for almost all the traits studied. The hybrids, KULLAKAR $\times$ IR 36 and ASHWANI $\times$ ADT 37 were recorded with high per se performance and highly significant sca effects for grain yield and its component traits were found to be suitable for heterosis breeding. The crosses viz., ADT 39 $\times$ ADT 45 and ASHWANI $\times$ ADT 45 were identified with high mean and non significant sca effects and could be exploited for recombination breeding. 
Table.1 Analysis of variance for combining ability analysis in rice

\begin{tabular}{|c|c|c|c|c|c|c|c|c|c|c|c|c|}
\hline \multirow{2}{*}{$\begin{array}{l}\text { Source of } \\
\text { variation }\end{array}$} & & \multicolumn{11}{|c|}{ MSS } \\
\hline & df & $\begin{array}{l}\text { Days to } 50 \\
\text { per cent } \\
\text { flowering } \\
\text { (days) }\end{array}$ & $\begin{array}{l}\text { Plant } \\
\text { height at } \\
\text { maturity } \\
(\mathrm{cm})\end{array}$ & $\begin{array}{l}\text { Number of } \\
\text { tillers per } \\
\text { plant }\end{array}$ & $\begin{array}{c}\text { Number of } \\
\text { productive } \\
\text { tillers per } \\
\text { plant }\end{array}$ & $\begin{array}{c}\text { Panicle } \\
\text { length } \\
\text { (cm) }\end{array}$ & $\begin{array}{l}\text { Number of } \\
\text { grains per } \\
\text { panicle }\end{array}$ & $\begin{array}{c}\text { Kernel } \\
\text { Length } \\
(\mathrm{mm})\end{array}$ & $\begin{array}{c}\text { Kernel } \\
\text { Breadth } \\
(\mathbf{m m})\end{array}$ & $\begin{array}{l}\text { Kernel } \\
\text { L / B } \\
\text { ratio }\end{array}$ & $\begin{array}{l}\text { Hundred } \\
\text { grain } \\
\text { weight (g) }\end{array}$ & $\begin{array}{l}\text { Grain } \\
\text { yield per } \\
\text { plant }(\mathrm{g})\end{array}$ \\
\hline Replication & 2 & 5.18 & 0.63 & 1.88 & 0.74 & 3.47 & 23.65 & 0.0025 & 0.0069 & 0.019 & 0.0084 & 0.74 \\
\hline Genotype & 43 & $483.33 * *$ & $750.28 * *$ & $350.43 * *$ & $253.63 * *$ & $68.10^{* *}$ & $3837.07 * *$ & $1.74 * *$ & $0.51 * *$ & $0.65 * *$ & $0.28 * *$ & $178.62 * *$ \\
\hline Cross & 31 & $461.18 * *$ & $537.45 * *$ & $274.88 * *$ & $224.00 * *$ & $77.50 * *$ & $2347.66^{* *}$ & $1.50 * *$ & $0.38 * *$ & $0.45^{* *}$ & $0.26 * *$ & $174.09 * *$ \\
\hline Line & 7 & $1871.71 * *$ & $1393.16^{* *}$ & $598.87 * *$ & $599.80 * *$ & $136.81^{* *}$ & $2561.64 * *$ & $4.48 * *$ & $0.57 *$ & $0.17 *$ & $0.36^{*}$ & $301.06 * *$ \\
\hline Tester & 3 & $11.25^{* *}$ & $438.37 * *$ & $289.52 * *$ & $121.00^{* *}$ & $174.84 * *$ & $4189.16^{* *}$ & $0.41 *$ & $0.23^{*}$ & $0.59 * *$ & $0.60 * *$ & $222.32 * *$ \\
\hline $\mathbf{L} \times \mathbf{T}$ & 21 & $55.28 * *$ & $266.37 * *$ & $164.80 * *$ & $113.44 * *$ & $43.83 * *$ & $2013.26^{* *}$ & $0.66 * *$ & $0.33 * *$ & $0.53 * *$ & $0.18 * *$ & $124.88 * *$ \\
\hline Error & 86 & 3.46 & 0.66 & 3.20 & 2.54 & 2.70 & 9.76 & 0.0008 & 0.0031 & 0.01 & 0.0039 & 0.57 \\
\hline \multicolumn{2}{|c|}{ GCA Variance } & 8.06 & 5.38 & 2.18 & 2.19 & 0.66 & 6.64 & 0.016 & 0.0008 & 0.0015 & 0.0016 & 0.97 \\
\hline \multicolumn{2}{|c|}{ SCA Variance } & 17.27 & 88.57 & 53.86 & 36.96 & 13.71 & 667.83 & 0.22 & 0.11 & 0.17 & 0.06 & 41.43 \\
\hline \multicolumn{2}{|c|}{ GCA/SCA Variance } & 0.46 & 0.06 & 0.04 & 0.06 & 0.04 & 0.009 & 0.07 & 0.007 & 0.008 & 0.02 & 0.02 \\
\hline
\end{tabular}

*** Significant at 5 and 1 per cent respectively. 
Table.2 General combining effects of parents for different traits in rice genotypes

\begin{tabular}{|c|c|c|c|c|c|c|c|c|c|c|c|}
\hline & $\begin{array}{c}\text { Days to } 50 \text { per } \\
\text { cent flowering } \\
\text { (days) }\end{array}$ & $\begin{array}{c}\text { Plant } \\
\text { height at } \\
\text { maturity } \\
(\mathrm{cm})\end{array}$ & $\begin{array}{c}\text { Number of } \\
\text { tillers per } \\
\text { plant }\end{array}$ & $\begin{array}{c}\text { Number of } \\
\text { productive } \\
\text { tillers per } \\
\text { plant }\end{array}$ & $\begin{array}{l}\text { Panicle } \\
\text { length } \\
\text { (cm) }\end{array}$ & $\begin{array}{c}\text { Number of } \\
\text { grains per } \\
\text { panicle }\end{array}$ & $\begin{array}{l}\text { Kernel } \\
\text { length }\end{array}$ & $\begin{array}{r}\text { Kernel } \\
\text { breadth }\end{array}$ & $\begin{array}{c}\text { Kernel } \\
\text { L/B } \\
\text { ratio }\end{array}$ & $\begin{array}{c}\text { Hundred } \\
\text { grain } \\
\text { weight (g) }\end{array}$ & $\begin{array}{c}\text { Grain } \\
\text { yield per } \\
\text { plant }(g)\end{array}$ \\
\hline \multicolumn{12}{|c|}{ Lines } \\
\hline ADT-43 & $-6.99 * *$ & $16.56 * *$ & $-13.46^{* *}$ & $-7.65 * *$ & $1.41 * *$ & $-13.06 * *$ & $0.08 * *$ & $0.08 * *$ & $-0.08 * *$ & $-0.05 * *$ & $-6.19 * *$ \\
\hline NLR-34449 & $-11.79 * *$ & $11.99 * *$ & -0.39 & $-4.90 * *$ & -0.76 & $4.80 * *$ & $-0.83 * *$ & $-0.05 * *$ & $-0.09 * *$ & $-0.05 *$ & $-0.82 * *$ \\
\hline $\begin{array}{l}\text { AKSHAYA } \\
\text { (BPT 2231) }\end{array}$ & $16.35 * *$ & $-4.54 * *$ & $-1.59 * *$ & $-5.71 * *$ & -0.91 & $-7.68 * *$ & $-1.04 * *$ & $-0.49 * *$ & -0.06 & $-0.12 * *$ & $-4.07 * *$ \\
\hline PONNI & $9.96 * *$ & $3.87 * *$ & $-4.39 * *$ & $-2.64 * *$ & $5.44 * *$ & $-5.02 * *$ & $0.06 * *$ & $-0.07 * *$ & 0.05 & $-0.20 * *$ & $2.69 * *$ \\
\hline ADT-39 & $4.10 * *$ & $-2.80 * *$ & $3.70 * *$ & $2.66^{* *}$ & $4.08 * *$ & $22.63 * *$ & $0.23^{* *}$ & $0.11 * *$ & $0.09 * *$ & $-0.13 * *$ & $-4.38 * *$ \\
\hline KULLAKAR & $-15.72 * *$ & $-16.82 * *$ & $10.52 * *$ & $13.85 * *$ & $-4.00 * *$ & $10.68 * *$ & $0.39 * *$ & $0.21 * *$ & $-0.16^{* *}$ & $0.29 * *$ & $5.27 * *$ \\
\hline $\begin{array}{c}\text { ASHWANI } \\
\text { (IC248372) }\end{array}$ & $-9.46^{* *}$ & $-8.25 * *$ & $4.55^{* *}$ & $5.30 * *$ & $-3.06 * *$ & $9.86^{* *}$ & $0.50 * *$ & $0.09 * *$ & $0.22 * *$ & $0.23 * *$ & $8.09 * *$ \\
\hline GIRI & $13.56^{* *}$ & -0.02 & $1.07 *$ & -0.91 & -2.19 & $-22.20 * *$ & $0.61 * *$ & 0.12 & 0.03 & 0.03 & $-0.59 * *$ \\
\hline $\begin{array}{c}\text { S.E. (GCA } \\
\text { effects for line) }\end{array}$ & 0.53 & 0.33 & 0.51 & 0.46 & 0.47 & 0.90 & 0.01 & 0.01 & 0.02 & 0.02 & 0.21 \\
\hline \multicolumn{12}{|c|}{ Testers } \\
\hline ADT-37 & $0.60 *$ & $-5.51 * *$ & $-3.89 * *$ & $-0.93 * *$ & 0.31 & $7.99 * *$ & $0.02 * *$ & $0.06^{* *}$ & $-0.12 * *$ & $0.22 * *$ & $2.47 * *$ \\
\hline ADT-45 & $-1.21 * *$ & $0.50 * *$ & $1.78 * *$ & $-2.30 * *$ & $-2.49 * *$ & $16.25^{* *}$ & $0.18 * *$ & $-0.14 * *$ & $0.21 * *$ & $-0.15 * *$ & $2.79 * *$ \\
\hline IR-36 & $0.58 *$ & $4.92 * *$ & $3.86 * *$ & $2.99 * *$ & $-1.47 * *$ & $13.12 * *$ & $-0.10 * *$ & $0.09 * *$ & $-0.13 * *$ & $-0.06 * *$ & $-2.45 * *$ \\
\hline IR-20 & 0.03 & 0.10 & $-1.74 * *$ & 0.24 & $3.66 * *$ & $-4.87 * *$ & $-0.09 * *$ & -0.01 & 0.04 & -0.02 & $-2.81 * *$ \\
\hline $\begin{array}{l}\text { S.E. (GCA } \\
\text { effects for } \\
\text { testers }\end{array}$ & 0.37 & 0.23 & 0.36 & 0.32 & 0.33 & 0.63 & 0.01 & 0.01 & 0.02 & 0.01 & 0.15 \\
\hline
\end{tabular}

*** Significant at 5 and 1 per cent respectively S.E.- Standard Error 
Table.3 Specific combining ability effects of hybrids for different traits in rice genotypes

\begin{tabular}{|c|c|c|c|c|c|c|c|c|c|c|c|}
\hline Cross & $\begin{array}{l}\text { Days to } 50 \text { per } \\
\text { cent flowering } \\
\text { (days) }\end{array}$ & $\begin{array}{l}\text { Plant height } \\
\text { at maturity } \\
(\mathrm{cm})\end{array}$ & $\begin{array}{c}\text { Number of } \\
\text { tillers per } \\
\text { plant }\end{array}$ & $\begin{array}{c}\text { Number of } \\
\text { productive tillers } \\
\text { per plant }\end{array}$ & $\begin{array}{c}\text { Panicle } \\
\text { length } \\
\text { (cm) }\end{array}$ & $\begin{array}{l}\text { Number of } \\
\text { grains per } \\
\text { panicle }\end{array}$ & $\begin{array}{l}\text { Kernel } \\
\text { length }\end{array}$ & $\begin{array}{l}\text { Kernel } \\
\text { breadth }\end{array}$ & $\begin{array}{c}\text { Kernel } \\
\text { L/B } \\
\text { ratio }\end{array}$ & $\begin{array}{l}\text { Hundred } \\
\text { grain } \\
\text { weight }(g)\end{array}$ & $\begin{array}{l}\text { Grain } \\
\text { yield per } \\
\text { plant (g) }\end{array}$ \\
\hline $\begin{array}{c}\text { ADT } 43 x \\
\text { ADT } 37\end{array}$ & -0.32 & $-2.74 * *$ & $8.37 * *$ & $1.97 *$ & $-4.08 * *$ & $9.26 * *$ & $0.25^{* *}$ & $0.36^{* *}$ & $-0.22 * *$ & $-0.18 * *$ & 0.81 \\
\hline $\begin{array}{c}\text { ADT } 43 x \\
\text { ADT } 45\end{array}$ & $-4.27 * *$ & $-5.19 * *$ & $-4.52 * *$ & -1.79 & $-3.76 * *$ & -3.12 & $-0.54 * *$ & $0.16^{* *}$ & $-0.73 * *$ & $0.08 *$ & $1.76 * *$ \\
\hline$\underset{36}{\text { ADT }} \underset{33}{x}$ IR & -0.10 & $-1.30 * *$ & -0.28 & -0.63 & $4.84 * *$ & $-12.69 * *$ & $0.27 * *$ & $-0.29 * *$ & $0.36 * *$ & 0.00 & -0.70 \\
\hline $\begin{array}{c}\text { ADT } 43 \times \text { IR } \\
20\end{array}$ & $4.69 * *$ & $9.23 * *$ & $-3.56 * *$ & 0.45 & $2.99 * *$ & $6.55^{* *}$ & $0.27 * *$ & $-0.58 * *$ & $0.59 * *$ & $0.15^{* *}$ & $-1.86^{* *}$ \\
\hline $\begin{array}{c}\text { NLR } 34449 x \\
\text { ADT } 37\end{array}$ & 0.11 & $-7.11 * *$ & $5.30 * *$ & $6.46 * *$ & 0.68 & $9.08 * *$ & $-0.70 * *$ & $-0.50 * *$ & $0.24 * *$ & -0.06 & $3.11 * *$ \\
\hline $\begin{array}{l}\text { NLR } 34449 x \\
\text { ADT } 45\end{array}$ & -1.68 & $-3.52 * *$ & $4.03 * *$ & $7.29 * *$ & $-2.28 *$ & -2.86 & $0.14^{* *}$ & $-0.24 * *$ & -0.08 & $0.33 * *$ & $-1.13 *$ \\
\hline $\begin{array}{l}\text { NLR } 34449 x \\
\quad \text { IR } 36\end{array}$ & $2.35^{*}$ & $10.28 * *$ & $-3.25 * *$ & $-9.70 * *$ & 0.07 & -1.89 & $0.16^{* *}$ & $0.28 * *$ & $0.15^{*}$ & $-0.24 * *$ & $-4.60 * *$ \\
\hline $\begin{array}{l}\text { NLR } 34449 x \\
\quad \text { IR } 20\end{array}$ & 0.77 & $-2.92 * *$ & $-6.08 * *$ & $-4.05^{* *}$ & 1.53 & $-4.34 *$ & $-0.58 * *$ & $0.47 * *$ & $-0.77 * *$ & -0.03 & $2.62 * *$ \\
\hline $\begin{array}{c}\text { AKSHYA } x \\
\text { ADT } 37\end{array}$ & -1.16 & $2.33 * *$ & $-3.98 * *$ & $-6.96 * *$ & -1.72 & $24.87 * *$ & $-0.47 * *$ & $0.35 * *$ & $-0.57 * *$ & $0.24 * *$ & -0.43 \\
\hline $\begin{array}{c}\text { AKSHYA } x \\
\text { ADT } 45\end{array}$ & $-3.27 * *$ & $10.92 * *$ & $4.47 * *$ & $4.53 * *$ & -1.36 & $-15.78 * *$ & $0.28 * *$ & $0.09 * *$ & 0.04 & $-0.43 * *$ & $3.85 * *$ \\
\hline $\begin{array}{c}\text { AKSHYA } x \\
\text { IR } 36\end{array}$ & $3.19 * *$ & $-7.38 * *$ & 1.30 & 0.55 & -0.62 & $-20.71 * *$ & $-0.03 *$ & $-0.15 * *$ & 0.10 & -0.00 & $-4.24 * *$ \\
\hline $\begin{array}{c}\text { AKSHYA } x \\
\text { IR } 20\end{array}$ & 1.23 & $-5.87 * *$ & -1.79 & $1.89 *$ & $3.70 * *$ & $11.62 * *$ & $0.23 * *$ & $-0.28 * *$ & $0.42 * *$ & $0.12^{* *}$ & $4.36 * *$ \\
\hline $\begin{array}{l}\text { PONNI x } \\
\text { ADT } 37\end{array}$ & 0.77 & $-2.30 * *$ & $2.30 *$ & $1.83^{*}$ & 1.20 & $44.56^{* *}$ & $0.16 * *$ & $-0.29 * *$ & $0.50 * *$ & $0.34 * *$ & $0.87 *$ \\
\hline $\begin{array}{l}\text { PONNI } x \\
\text { ADT } 45\end{array}$ & -0.66 & $6.61 * *$ & $-16.93^{* *}$ & $-11.37 * *$ & $2.19 *$ & $5.07 * *$ & $-0.77 * *$ & $-0.21 * *$ & -0.07 & $-0.26 * *$ & $-4.32 * *$ \\
\hline
\end{tabular}




\begin{tabular}{|c|c|c|c|c|c|c|c|c|c|c|c|}
\hline $\begin{array}{c}\text { PONNI x IR } \\
36\end{array}$ & $-4.77 * *$ & $2.70 * *$ & $8.50 * *$ & $10.78 * *$ & $-5.74 * *$ & $10.23 * *$ & $0.09 * *$ & $0.13 * *$ & $-0.14 *$ & $-0.19 * *$ & $-4.28 * *$ \\
\hline $\begin{array}{c}\text { PONNI } x \text { IR } \\
20\end{array}$ & $4.66^{* *}$ & -0.60 & $6.13 * *$ & -1.24 & $-2.05^{*}$ & $-17.75^{* *}$ & $0.53 * *$ & $0.36^{* *}$ & $-0.29 * *$ & $0.11 * *$ & $7.72 * *$ \\
\hline $\begin{array}{l}\text { ADT-39 x } \\
\text { ADT } 37\end{array}$ & 0.87 & $8.00 * *$ & $-9.92 * *$ & $-7.48 * *$ & 0.61 & $-42.77 * *$ & $0.13 * *$ & -0.06 & $0.15 *$ & $-0.13 * *$ & $-3.60 * *$ \\
\hline $\begin{array}{c}\text { ADT-39 x } \\
\text { ADT } 45\end{array}$ & $11.94 * *$ & 0.21 & 1.18 & 0.46 & $-4.36 * *$ & 2.45 & 0.02 & 0.03 & 0.06 & 0.02 & 0.31 \\
\hline $\begin{array}{c}\text { ADT-39 x IR } \\
36\end{array}$ & $3.77 * *$ & $18.57 * *$ & $-6.74 * *$ & -1.41 & $-4.10 * *$ & $-17.02 * *$ & $-0.35 * *$ & $-0.08^{*}$ & $-0.12 *$ & $-0.07 *$ & $-15.77 * *$ \\
\hline $\begin{array}{c}\text { ADT-39 } x \text { IR } \\
20\end{array}$ & -1.90 & $6.95 * *$ & $2.82 * *$ & 0.39 & $2.35 *$ & $15.23 * *$ & $-0.05 * *$ & $0.11^{* *}$ & $-0.27 * *$ & $0.13 * *$ & $-3.62 * *$ \\
\hline $\begin{array}{c}\text { KULLAKAR } \\
\text { x ADT } 37\end{array}$ & $3.48 * *$ & $-1.35 * *$ & $-6.11 * *$ & 0.08 & -1.73 & $-23.44 * *$ & $0.37 * *$ & 0.01 & -0.03 & $-0.14 * *$ & -4.97 \\
\hline $\begin{array}{c}\text { KULLAKAR } \\
\text { x ADT } 45\end{array}$ & -0.32 & $4.24 * *$ & $5.10 * *$ & $-4.17 * *$ & 1.60 & $-25.79 * *$ & $-0.36 * *$ & $0.11 * *$ & $-0.25 * *$ & 0.06 & -9.19 \\
\hline $\begin{array}{c}\text { KULLAKAR } \\
\text { x IR } 36\end{array}$ & $-5.80 * *$ & $-21.56 * *$ & $13.84 * *$ & $5.87 * *$ & $6.59 * *$ & $70.31 * *$ & $1.12 * *$ & $0.52 * *$ & $0.56 * *$ & $0.36^{* *}$ & $17.47 * *$ \\
\hline $\begin{array}{c}\text { KULLAKAR } \\
\text { x IR } 20\end{array}$ & $-4.03 * *$ & $-3.59 * *$ & $2.86^{* *}$ & -1.78 & $-2.06 *$ & $-21.09 * *$ & $-0.15 * *$ & $-0.45 * *$ & $0.36^{* *}$ & $-0.27 * *$ & $-3.31 * *$ \\
\hline $\begin{array}{c}\text { ASHWANI } x \\
\text { ADT } 37\end{array}$ & $-4.24 * *$ & $-13.62 * *$ & $6.55 * *$ & $8.50 * *$ & $6.11 * *$ & $18.65 * *$ & $0.27 * *$ & $0.18 * *$ & $0.16^{* *}$ & $0.37 * *$ & $12.98 * *$ \\
\hline $\begin{array}{l}\text { ASHWANI } x \\
\text { ADT } 45\end{array}$ & -0.23 & 0.35 & 0.73 & 1.18 & $1.93 *$ & 1.89 & 0.01 & 0.04 & 0.01 & 0.06 & 0.36 \\
\hline $\begin{array}{c}\text { ASHWANI } x \\
\text { IR } 36\end{array}$ & 0.88 & $-3.56 * *$ & $-2.95 * *$ & $2.27 *$ & $2.41 *$ & $-12.09 * *$ & $-0.26 * *$ & $-0.13 * *$ & $0.25^{* *}$ & $-0.17 * *$ & -0.01 \\
\hline $\begin{array}{c}\text { ASHWANI } x \\
\text { IR } 20\end{array}$ & $-4.43 * *$ & $2.24 * *$ & $-3.40 * *$ & 1.44 & $-4.95 * *$ & $9.63 * *$ & $-0.23 * *$ & $0.24 * *$ & $-0.42 * *$ & $-0.20 * *$ & $-2.57 * *$ \\
\hline $\begin{array}{c}\text { GIRI } \times \text { ADT } \\
\mathbf{3 7}\end{array}$ & -0.85 & $-1.06^{*}$ & $4.67 * *$ & $1.84^{*}$ & -1.07 & 0.57 & $0.22 * *$ & $-0.20 * *$ & -0.06 & -0.06 & $1.28 * *$ \\
\hline $\begin{array}{c}\text { GIRI } x \\
45\end{array}$ & -1.52 & $7.51 * *$ & $3.48 * *$ & $-4.16^{* *}$ & 1.64 & -2.65 & $-0.21 * *$ & $-0.18 * *$ & -0.04 & $-0.18 * *$ & $0.87 *$ \\
\hline $\begin{array}{c}\text { GIRI } x \\
36\end{array}$ & 1.82 & $2.24 * *$ & -1.24 & $-5.91 * *$ & 0.95 & $-16.15^{* *}$ & -0.02 & $0.08^{*}$ & $-0.28 * *$ & $0.25^{* *}$ & $2.11 * *$ \\
\hline GIRI x IR 20 & 0.55 & $-8.69 * *$ & -0.67 & $2.90 * *$ & -1.52 & 0.15 & $0.22 * *$ & $0.14 * *$ & $0.38 * *$ & -0.01 & -3.34 \\
\hline $\begin{array}{l}\text { SCA effects } \\
\text { for hybrids }\end{array}$ & 1.07 & 1.46 & 1.03 & 0.92 & 0.94 & 1.80 & 0.01 & 0.03 & 0.02 & 0.03 & 0.43 \\
\hline
\end{tabular}

*** Significant at 5 and 1 per cent respectively S.E.- Standard Error 
References

Asadur Rahaman, 2016 Study of nature and magnitude of gene action in hybrid rice (Oryza sativa L.) through experiment of line $\mathrm{x}$ tester mating design. Intl. J. of App. Res., 2(2):405410.

Cockerham, C. C. 1961. Implication of genetic variances in a hybrid breeding programme. Crop Science, 8: 720 722.

Gayathri. 2015. Studies on genetic divergence and combining ability in rice (Oryza sativa L.) under coastal salinity. M.Sc(Ag) Thesis, Annamalai Univ., Annamalainagar, India.

Griffing B (1956) Concept of general and specific combining ability in relation to diallel crossing system, Australian J. of Bio. Sci. 9: 463-493.

Kempthorne, O. 1957. An introduction to genetic statistics, John Wiley and Sons, Inc., New York.

Nainu A. J., K. Palaniraja and S. Vennila 2016. Combining ability studies for development of new hybrids in rice (oryza sativa 1.) under coastal saline condition. Indo - Asian J. of
Multidisciplinary Res. (IAJMR) 2(3);643 - 648.

Pradhan, S.K. and S. Singh. 2008. Combining ability and gene action analysis for morphological and quality traits in basmati rice. Oryza, 45(3): 193-197.

Sanghera, G.S. and Hussain, W. 2012. Heterosis and combining ability estimates using line $\mathrm{x}$ tester analysis to develop rice hybrids for temperature conditions. Not. Sci. Bio. 4(3): 131142.

Singh, R.K and B.D. Chaudhury. 1985. Biometrical methods in quantitative genetic analysis. Kalyani publishers, New Delhi.

Upadhyay. M.N, and H.K. Jaiswal. 2015. Combining ability analysis for yield and earliness in hybrid rice (Oryza sativa L.). Asian J. of Crop Sci., 7(1): 81-86.

Zeinab Montazer, Nadal Babaeian Jelodar, Nadali Bagheri. 2014. Genetic dissection of some important agronomic traits in rice using line $\mathrm{x}$ tester method. International Journal of Advanced Biological and Biomedical Research. 2(1):181-191.

\section{How to cite this article:}

Thirumalai, R., K. Palaniraja and Vennila, S. 2018. Yield Response of Rice Genotypes for Gene Action under Coastal Saline Condition. Int.J.Curr.Microbiol.App.Sci. 7(04): 3353-3360. doi: https://doi.org/10.20546/ijcmas.2018.704.379 